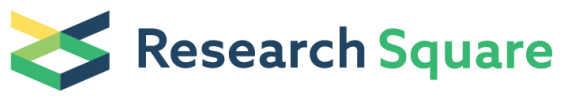 \\ Preprints are preliminary reports that have not undergone peer review. \\ They should not be considered conclusive, used to inform clinical practice, or referenced by the media as validated information.
}

\section{The COVID-19 Pandemic Accelerated Publication of Medical Articles}

\section{Lu Wang}

Tongji Hospital of Tongji Medical College of Huazhong University of Science and Technology

\section{Xinyi Chen}

Tongji Hospital of Tongji Medical College of Huazhong University of Science and Technology

Yinan Sun

Tongji Hospital of Tongji Medical College of Huazhong University of Science and Technology

Han Yin

Tongji Hospital of Tongji Medical College of Huazhong University of Science and Technology

Long Li

Tongji Hospital of Tongji Medical College of Huazhong University of Science and Technology

Bo Liu

Tongji Hospital of Tongji Medical College of Huazhong University of Science and Technology

\section{Weiheng Zhao}

Tongji Hospital of Tongji Medical College of Huazhong University of Science and Technology

\section{Bili Wu}

Tongji Hospital of Tongji Medical College of Huazhong University of Science and Technology

\section{Chaofan Liu}

Tongji Hospital of Tongji Medical College of Huazhong University of Science and Technology

\section{Qin Huang}

Tongji Hospital of Tongji Medical College of Huazhong University of Science and Technology

Xianglin Yuan ( $\nabla$ yuanxianglin@hust.edu.cn)

Tongji Hospital of Tongji Medical College of Huazhong University of Science and Technology

\section{Research}

Keywords: COVID-19, Clinical trial, Medical articles

Posted Date: April 8th, 2021

DOI: https://doi.org/10.21203/rs.3.rs-390718/v1

License: (1) This work is licensed under a Creative Commons Attribution 4.0 International License. Read Full License 


\section{Abstract}

\section{Background}

Experimental biological research and clinical trials that relied on the healthcare system, access to public laboratory equipment, and adequate space were particularly affected by the COVID-19 pandemic and mobility restrictions. The study is to analyze the influence of COVID-19 on medical research by exploring the clinical trials and articles published by medical researchers worldwide during the COVID-19 pandemic.

\section{Methods}

Routinely conducted, deferred, and terminated clinical trial statistics from the Cancer Center of Tongji Hospital were collected during the COVID-19 pandemic. The number of global medical articles published in 2020 and those from the previous five years were analyzed according to the PubMed database. To conduct subgroup analyses, the articles were classified according to type and research field.

\section{Results}

The total number of publications per month in 2020 increased compared to the number of articles published in 2019. However, a decrease in the number of clinical trials was noted. Reviews and research papers increased by $7.28 \%$ and $20.60 \%$, respectively. The number of clinical trial published decreased by $62.29 \%$. The proportion of cancer-related publications (38.44\% vs. $44.79 \%)$ decreased, whereas the proportion of immunology, pulmonology, and emergency publications $(19.23 \%$ vs. $17.48 \%)$ increased. In the Cancer Center of the Tongji Hospital in Wuhan during the COVID-19 pandemic, of the 46 clinical trials analyzed, 37 (80.40\%) were delayed, $8(17.40 \%)$ were remote, and $1(2.20 \%)$ was routine.

\section{Conclusions}

The COVID-19 pandemic had promoted the publication of medical research articles especially those related to the immunology, pulmonology, and emergency medicine. It had a pronounced negative impact on the execution and publication of clinical trials.

\section{Introduction}

The first case of coronavirus disease (COVID-19), which was caused by the severe acute respiratory syndrome coronavirus 2 (SARSCoV-2), was reported in December 2019 [1, 2]. The World Health Organization (WHO) officially declared the outbreak of COVID-19 as a pandemic on March 11, 2020 [3]. As of 1st January 2021, more than 81 million people had been infected and more than 1.8 million people had died from COVID-19. As a result of this pandemic, the entire world had experienced unprecedented public health crises along with exceptional social challenges. COVID-19 had overburdened many healthcare systems $[4,5]$. Even though certain vaccines and drugs had been approved to prevent or treat COVID-19, there was currently no specific antiviral treatment for the disease. Therefore, tracking the transmission route of COVID-19 was critical for controlling its spread. Most countries had taken active measures to curb the spread of COVID-19 such as home isolation, telecommuting, reducing the number of indoor staff, or providing internet-based medical services [6-8]. A survey reported that approximately $60 \%$ of the labor force in the United States worked from home during the pandemic [9]. More than 3.5 billion individuals had been confined to their homes and, as a result, several million had worked remotely [10]. These isolation measures had led to significant changes in social relationships, the mode of medical care, and the pace of economic development. The restrictions of movement and limitations on personal contacts had severely disrupted the daily routines of people worldwide [11].

Due to the variety of restrictions placed on people's daily lives, medical research had also been significantly affected. Experimental biological research and clinical trials that relied on the healthcare system, access to public laboratory equipment, and/or adequate space were particularly affected. Because of mobility restrictions, many new clinical trials encountered difficulties in recruiting subjects and ongoing clinical trials experienced poor follow-up rates [12]. A recent analysis showed that approximately $60 \%$ of clinical trial investigators reported a significant negative impact on protocol scheduled visits from the COVID-19 pandemic. This resulted in protocol deviations, unresolved queries, and, eventually, low-quality data entry [13]. The research and clinical trials in the field of oncology had increased exponentially in recent years [14]. However, since the COVID-19 outbreak, the research of non-

Page 2/10 
COVID-19 diseases, including cancer, has been considered as "non-urgent" and had been postponed [15]. These sudden interruptions had inevitably impacted cancer research [16]. Therefore, we conducted this research to present an in-depth analysis of the 2020 publication of medical papers worldwide and the clinical trials at a research center in Wuhan. The analysis was conducted to explore the impact of the COVID-19 pandemic and the resulting prevention measures on the conduction of scientific medical research.

\section{Methods}

\section{Research strategy}

Utilizing the PubMed database, medical articles published from January 1, 2020, to December 31, 2020 were included. Reviews, research papers, and clinical trials were screened. Article categories were classified according to fields such as COVID-19, immunology, pulmonology, emergency medicine, and cancer-associated diseases. The articles published per month in each subtype within the prior five years were analyzed and compared with the articles published in 2020 . We also summarized the actual status changes of the clinical trials conducted at the Cancer Center of the Tongji Hospital during the pandemic. Routine, referred, remote, or discontinued trails were reviewed to analyze the impact of the pandemic on the execution of clinical trials for oncology.

\section{Indicators-outcomes}

The number of published articles in 2020 and the prior five years were analyzed with regard to research subtype. These included reviews, original research, and clinical trials in various fields such as immunology, pulmonology, cancer, or emergency medicine. We also analyzed the clinical research of the Cancer Center of the Tongji Hospital in Wuhan. We gathered statistics related to the actual status of research (normal, referred, remote, or stopped) that was being conducted. The average growth rate in five years is defined as an average of the number of growth rates published in 2015-2019. The highest/lowest growth rate in five years is defined as the highest/lowest growth rate of articles published in one year from to 2015-2019.

\section{Statistical analysis}

Descriptive research and analyses were performed according to each research subtype. Articles published in various years and in various research fields, plus the additional clinical trials conducted the research center, were analyzed. The number of articles published in each month in 2015-2020 was compared using the Mann-Whitney nonparametric test. Statistical analysis and mapping were conducted using GraphPad Prism 5.0 (GraphPad, San Diego, CA, USA) and SPSS software (version 16.0, SPSS Inc., Chicago, IL). A 2-sided statistically significance threshold was set at $P<0.05$.

\section{Results}

Based on data extracted from PubMed, a total of 594,105 medical-related articles, including reviews $(60,686,10.21 \%)$, research papers $(517,942,87.18 \%)$, and others $(15,477,2.61 \%)$, were published between January 1, 2020 and December 31, 2020. Clinical trials represented $1.14 \%$ of these articles. As the severity and prognosis of COVID-19 may be related to tumors, immunology, pulmonology, and emergency medicine, we classified the articles according to these fields. There were 87,982 articles related to COVID-19, 227,745 to tumors, 85,053 to immunology, 58,416 to pulmonology, and 114,272 to emergency medicine, as shown in Table 1. 
Table 1

The number of published articles according to type from 2015 to 2020.

\begin{tabular}{|llllll|}
\hline & Article & Review & Original Research & Clinical Trial & Others \\
\hline 2020 & 594,105 & 60,686 & 517,942 & 6,791 & 15,477 \\
\hline 2019 & 505,916 & 56,567 & 429,475 & 18,008 & 19,874 \\
\hline 2018 & 472,992 & 58,546 & 395,877 & 19,110 & 18,569 \\
\hline 2017 & 448,400 & 60,030 & 371,095 & 19,101 & 17,275 \\
\hline 2016 & 427,310 & 57,111 & 354,676 & 18,603 & 15,523 \\
\hline 2015 & 390,176 & 50,551 & 324,489 & 18,039 & 15,136 \\
\hline
\end{tabular}

\section{The Outbreak Of Covid-19 Increased Article Publication In 2020}

A decrease in the number of clinical trial publications was noted. However, the total number of articles published in 2020 showed an increasing trend compared with the number of articles published in 2019. Reviews and research papers increased by $7.28 \%$ and $20.60 \%$, respectively, in 2020 . However, the number of clinical trial results published decreased by $62.29 \%$, as shown in Fig. $1 \mathrm{~A}$. Regarding the proportion of publications recorded for 2020 and 2019, the proportion of cancer-related publications ( $38.44 \%$ vs. $44.79 \%$ ) decreased; however, the proportion of immunology-related publications (14.32\% vs $13.99 \%)$, pulmonology-related publications ( $9.83 \%$ vs. $6.96 \%)$, and emergency-related publications (19.23\% vs. $17.48 \%)$ increased.

\section{The influence of the COVID-19 pandemic on trends of published articles}

A further comparative analysis of the growth trend of medical research published in the last five years showed that the total number of articles increased annually from 2015 to 2019 . The average annual growth was $28,933(21,090-37,134)$. The total number of articles growth in 2020/2019 was 88,774 . This is 3.07 times the average annual growth from 2015 to 2019 and 2.39 times the highest growth from 2015 to 2019. Analyzed with articles from 2015 to 2020 per month, as shown in Table 2, the total number of articles growth published in 2020/2019 increased compared with the number of growth in $2019 / 2018(P<0.01)$. The others had no statistical significance except for 2016 to $2017(P<0.05)$ shown in Fig. 2A. From 2015 to 2019, the average annual growth for immunology-related publications was 3,571 (2,503-5,543), an increase of 14,253 was noted in 2020 compared with 2019 which was 3.99 times the average annual growth in 2015-2019 and 2.57 times of the highest growth in 2015-2019. The average annual growth for pulmonology-related publications was 1,369 (991-1,747) from 2015 to 2019, an increase of 23,187 which was recorded for 2020 compared with 2019 which was 16.94 times the average annual growth of 2015-2019 and 13.27 times the highest growth of 2015-2019. The average annual increase for emergency-related publications was 5,248 (3,173-6,213) from 2015 to 2019 . This compared with 2019 is an increase of 25,820 that was recorded for 2020. This is 4.92 times the average annual growth in 20152019 and 4.16 times the highest growth in 2015-2019, as shown in Table 3. However, the opposite result was shown for clinical trials. The number of clinical trials published in 2015-2019 was stable and ranged between 18,039 in 2015 and 19,110 in 2018 . Although many new COVID-19-related clinical trials were conducted, only 6,791 (37.65\% of the lowest number of annual publications from 2015-2019) were published in 2020, as shown in Fig. 2B. 
Table 2

The number of published articles according to month from 2015 to 2020.

\begin{tabular}{|lllllllllllll|}
\hline & Jan. & Feb. & Mar. & Apr. & May & Jun. & Jul. & Aug. & Sep. & Oct. & Nov. & Dec. \\
\hline 2020 & 56,875 & 42,039 & 43,864 & 45,364 & 47,942 & 51,512 & 53,440 & 50,279 & 54,313 & 53,522 & 50,319 & 45,212 \\
\hline 2019 & 54,200 & 38,844 & 41,390 & 40,606 & 41,470 & 40,623 & 42,589 & 40,375 & 39,587 & 41,635 & 42,101 & 42,488 \\
\hline 2018 & 49,761 & 37,393 & 39,794 & 37,916 & 37,696 & 38,292 & 37,853 & 37,069 & 38,439 & 41,136 & 39,213 & 38,432 \\
\hline 2017 & 49,484 & 33,763 & 37,799 & 34,746 & 35,711 & 36,694 & 36,639 & 34,997 & 37,210 & 36,703 & 36,749 & 37,906 \\
\hline 2016 & 50,377 & 31,594 & 34,972 & 33,820 & 33,435 & 35,086 & 34,623 & 33,872 & 35,628 & 34,948 & 34,441 & 34,515 \\
\hline 2015 & 48,646 & 26,572 & 31,176 & 30,267 & 28,828 & 31,311 & 32,329 & 29,331 & 32,942 & 33,300 & 31,502 & 33,972 \\
\hline
\end{tabular}

Table 3

The number of published articles according to research field from 2015 to 2020.

\begin{tabular}{|lllll|}
\hline & Cancer & Immunology & Pulmonology & Emergency \\
\hline 2020 & 227,745 & 85,053 & 54,851 & 114,272 \\
\hline 2019 & 226,590 & 70,800 & 46,488 & 88,452 \\
\hline 2018 & 215,545 & 65,257 & 44,915 & 82,549 \\
\hline 2017 & 207,741 & 62,075 & 42,777 & 76,336 \\
\hline 2016 & 201,755 & 59,019 & 40,801 & 73,163 \\
\hline 2015 & 198,554 & 56,516 & 40,368 & 67,459 \\
\hline
\end{tabular}

COVID-19 had a negative impact on clinical trials conducted at the Cancer Center of Tongji Hospital in Wuhan

We analyzed the clinical trial data from a medical research center in Wuhan, the Cancer Center of Tongji Hospital, Tongji Medical College, Huazhong University of Science and Technology, as shown in Table 4. We found that 103 clinical trials were in progress as of February 27, 2020. Forty-seven of these had not enrolled patients or had not entered the data processing stage. According to the research plans, the patients in 56 projects were required to be visited and treated from January 2020 to February 2020 . Of the 46 clinical trials analyzed, $37(80.40 \%)$ were delayed, 8 (17.40\%) were remote, and $1(2.20 \%)$ was routine. Of the 44 clinical trials that involved drug use, 33 (75.00\%) were discontinued, 1 (2.20\%) was completed, and 10 (22.80\%) were continued. Of the 46 clinical trials under clinical supervision, 9 (19.60\%) were delayed, and 37 (80.40\%) were routine, shown in Fig. 1B. Clinical trials were seriously affected by the pandemic, mainly due to restrictions in follow-up, patient registration, and hospital access.

Table 4

Impact of the COVID-19 pandemic on clinical trials in the Cancer Center of Tongji Hospital in 2020.

\begin{tabular}{|lllll|}
\hline & Deferred & Remote & Normal & Finish \\
\hline Visit & 80.4 & 17.4 & 2.2 & - \\
\hline Clinical monitor & 19.6 & - & 80.4 & - \\
\hline Drug treatment & 71.8 & - & 21.8 & 2.2 \\
\hline
\end{tabular}

\section{Discussion}

In this study, we sought to explore the potential impact of the COVID-19 pandemic on the publication of medical articles. We obtained our results by comparing the number and category of published articles between 2015-2019 and 2020. We found a decrease in clinical trial publications, whereas reviews and original research showed an increase. Next, we investigated the 
proportion of publications in various fields. We found that the proportion of cancer-related publications decreased, whereas the proportion of immunology, pulmonology, and emergency-related publications increased. To further explain the underlying reasons for these article publication discrepancies, we also analyzed the clinical trial data of a medical research center in Wuhan, the Cancer Center of Tongji Hospital. The analysis showed that the clinical trials performed in the Cancer Center were markedly affected by the COVID-19 pandemic. We found that it was common for clinical trials to be delayed during the pandemic. This partially explains the decrease in clinical trial articles published in 2020. Collectively, the outbreak of COVID-19 increased the output of medical researchers worldwide, although there were differences between the various categories of articles.

Even though the proportion of tumor-related publications is declining, the COVID-19 pandemic may have greatly increased the publication of medical research. This is especially evident in the fields of immunology, pulmonology, and emergency medicine. The reasons may be a result of the following: first, the COVID-19 pandemic provided new hotspots and cross-cutting opportunities for medical research. Second, isolation measures and telecommuting provided sufficient time for researchers to organize data and publish papers. Third, cancer research was more dependent on the integration of instruments, equipment, the availability of research time, and the development of clinical trials. This resulted in a decline of the publication of clinical trials. We also noted that the possible reasons for the decline in the number of clinical trial publications during the pandemic were as follows. First, the higher demand for medical resources made it difficult for medical institutions to maintain a complete team and carry out the procedures for clinical trials. Second, patients were lost to follow-up due to COVID-19 isolation measures. Third, the inaccessibility of clinical trial drugs reduced the feasibility of clinical trial research, which led to the early termination of many clinical trials.

Other studies have shown that the COVID-19 pandemic has perturbed many aspects of clinical care, including clinical trials [17]. This has been threatening the integrity of clinical trials overall [18]. Due to the lack of on-site clinical research staff and the necessity of minimizing face-to-face contact with potential participants, many trials have suspended recruitment [19]. Consequently, there are currently many challenges associated with conducting clinical trials. These challenges include difficulties in registration as well as protocol treatment compliance due to the limited accessibility of participants and research staff $[20,21]$. These difficulties may also lead to inadequate outcome data that is required to fulfill sponsors' logistical trial obligations. These negative effects occurred often in oncology clinical trials resulting in lower enrollment rates [16]. Given the fact that cancer patients are more vulnerable and at high risk for contracting infections, they would have more serious complications from a potential COVID-19 infection [20,22, 23]. In addition, due to the urgency of developing effective therapeutics and vaccines for COVID-19, research on other diseases such as cancer is not considered a priority [15]. For example, the government of China suspended all clinical trials other than COVID-19 investigations in Wuhan, Hubei province [24]. The global situation from April to May was similar to that of China in February in terms of interruptions in clinical trials. These facts provide evidence for our results that clinical trial publications decreased during the COVID-19 pandemic in 2020. The data from the Cancer Center of our hospital's ongoing oncology clinical trials demonstrated the same tendency. This suggests that the COVID-19 pandemic indeed had a substantial impact on clinical trials, specifically oncology-related clinical trials. The decline in the proportion of cancer-related publications could be explained by this fact. In addition, the current pandemic has led to a great deal of changes in the work settings for most medical researchers. It has made remote working a necessity [25]. On the other hand, it has been demonstrated that remote working has a positive effect on work-life balance, which might enhance researchers' efficiency [26, 27]. For these reasons, many medical researchers could have been disengaged from their daily clinical work. Thus, more time was available to analyze data and write articles. Consequently, the reason the overall number of medical publications have increased becomes clear. This means that our results are consistent with those of previous studies.

The results of our study raised several issues, particularly the impact of the COVID-19 pandemic on medical publications and clinical trials. Systematic reviews are required to further assess the pandemic's global impact on the implementation of medical research and clinical trials. The impact of the COVID-19 pandemic on clinical practice could have profound effects on postpandemic study protocols. Adjustments and additional preparations are essential for medical researchers.

\section{Abbreviations}

COVID-19

Coronavirus disease 2019; SARS-CoV-2:Severe acute respiratory syndrome coronavirus-2; WHO:The World Health Organization. 


\section{Declarations}

\section{Acknowledgement}

\section{Authors' contributions:}

Concept and design: L. Wang, X.Y. Chen, Y.N. Sun, Q. Huang, X.L. Yuan.

Acquisition, analysis, or interpretation of data: H. Yin, L. Li, B. Liu, W.H. Zhao, B.L. Wu, C.F. Liu.

Drafting of the manuscript: L. Wang, X.Y. Chen.

Critical revision of the manuscript for important intellectual content: Q. Huang, X.L. Yuan.

Statistical analysis: L. Wang, X.Y. Chen.

Administrative, technical, or material support: H. Yin, L. Li, B. Liu, W.H. Zhao, B.L. Wu, C.F. Liu.

Supervision: Q. Huang, X.L. Yuan.

Funding: This article was supported by Tongji Hospital fund (2019YJJA14).

Competing interests: The authors have declared no conflicts of interest.

Consent for publication: Not applicable.

Ethics approval and consent to participate: Not applicable.

Availability of data and materials: The data underlying this article will be shared on reasonable request from the corresponding author.

\section{Author details}

Lu Wang, Email: wanglu@hust.edu.cn. Department of Oncology, Tongji Hospital, Tongji Medical College, Huazhong University of Science and Technology, Wuhan, Hubei, China.

Xinyi Chen, Email: chenxy1116@hust.edu.cn. Department of Oncology, Tongji Hospital, Tongji Medical College, Huazhong University of Science and Technology, Wuhan, Hubei, China.

Yinan Sun, Email: sunyinan@hust.edu.cn. Department of Cardiology, Tongji Hospital, Tongji Medical College, Huazhong University of Science and Technology, Wuhan, Hubei, China.

Han Yin, Email: hanyinoncogene@163.com. Department of Oncology, Tongji Hospital, Tongji Medical College, Huazhong University of Science and Technology, Wuhan, Hubei, China.

Long Li, Email: Ilregh@163.com. Department of Oncology, Tongji Hospital, Tongji Medical College, Huazhong University of Science and Technology, Wuhan, Hubei, China.

Bo Liu, Email: boliu888@hotmail.com. Department of Oncology, Tongji Hospital, Tongji Medical College, Huazhong University of Science and Technology, Wuhan, Hubei, China.

Weiheng Zhao, Email: zhaoweiheng319420@163.com. Department of Oncology, Tongji Hospital, Tongji Medical College, Huazhong University of Science and Technology, Wuhan, Hubei, China.

Bili Wu, Email: billywu2020@outlook.com. Department of Oncology, Tongji Hospital, Tongji Medical College, Huazhong University of Science and Technology, Wuhan, Hubei, China. 
Chaofan Liu, Email: Icf20101995@gmail.com. Department of Oncology, Tongji Hospital, Tongji Medical College, Huazhong University of Science and Technology, Wuhan, Hubei, China.

\section{References}

1. Zhu N, Zhang D, Wang W, Li X, Yang B, Song J, Zhao X, Huang B, Shi W, Lu R et al: A Novel Coronavirus from Patients with Pneumonia in China, 2019. N Engl J Med 2020, 382(8):727-733.

2. Wu F, Zhao S, Yu B, Chen YM, Wang W, Song ZG, Hu Y, Tao ZW, Tian JH, Pei YY et al: A new coronavirus associated with human respiratory disease in China. Nature 2020, 579(7798):265-269.

3. Mahase E: Covid-19: WHO declares pandemic because of "alarming levels" of spread, severity, and inaction. Bmj-Brit Med $J$ 2020, 368.

4. Ranney ML, Griffeth V, Jha AK: Critical Supply Shortages - The Need for Ventilators and Personal Protective Equipment during the Covid-19 Pandemic. N Engl J Med 2020, 382(18):e41.

5. Abbasi J: "Abandoned" Nursing Homes Continue to Face Critical Supply and Staff Shortages as COVID-19 Toll Has Mounted. JAMA 2020, 324(2):123-125.

6. Ng Y, Li Z, Chua YX, Chaw WL, Zhao Z, Er B, Pung R, Chiew CJ, Lye DC, Heng D et al: Evaluation of the Effectiveness of Surveillance and Containment Measures for the First 100 Patients with COVID-19 in Singapore - January 2-February 29, 2020. MMWR Morb Mortal Wkly Rep 2020, 69(11):307-311.

7. Dirlikov E, Fechter-Leggett E, Thorne SL, Worrell CM, Smith-Grant JC, Chang J, Oster AM, Bjork A, Young S, Perez AU et al: CDC Deployments to State, Tribal, Local, and Territorial Health Departments for COVID-19 Emergency Public Health Response United States, January 21-July 25, 2020. MMWR Morb Mortal Wkly Rep 2020, 69(39):1398-1403.

8. Elgendy MO, Abd Elmawla MN, Abdel Hamied AM, El Gendy SO, Abdelrahim MEA: COVID-19 patients and contacted person awareness about home quarantine instructions. Int J Clin Pract 2020:e13810.

9. Venkatesh V: Impacts of COVID-19: A research agenda to support people in their fight. Int J Inf Manage 2020, 55:102197.

10. Bouziri H, Smith DRM, Descatha A, Dab W, Jean K: Working from home in the time of COVID-19: how to best preserve occupational health? Occup Environ Med 2020, 77(7):509-510.

11. King AJ, Burke LM, Halson SL, Hawley JA: The Challenge of Maintaining Metabolic Health During a Global Pandemic. Sports Med 2020, 50(7):1233-1241.

12. Sathian B, Asim M, Banerjee I, Pizarro AB, Roy B, van Teijlingen ER, do Nascimento IJB, Alhamad HK: Impact of COVID-19 on clinical trials and clinical research: A systematic review. Nepal J Epidemiol 2020, 10(3):878-887.

13. Bouleftour W, Daguenet E, Tinquaut F, Magne N: Impact of COVID-19 Outbreak through Telemedicine Implementation on Data Reporting During Oncology Clinical Trials. Cancer Invest 2021, 39(1):15-20.

14. Tang J, Pearce L, O'Donnell-Tormey J, Hubbard-Lucey VM: Trends in the global immuno-oncology landscape. Nat Rev Drug Discov 2018, 17(11):783-784.

15. Ledford $\mathrm{H}$ : Coronavirus shuts down trials of drugs for multiple other diseases. Nature 2020, 580(7801):15-16.

16. Upadhaya S, Yu JX, Oliva C, Hooton M, Hodge J, Hubbard-Lucey VM: Impact of COVID-19 on oncology clinical trials. Nat Rev Drug Discov 2020, 19(6):376-377.

17. Rohr S, Arai H, Mangialasche F, Matsumoto N, Peltonen M, Raman R, Riedel-Heller SG, Sakurai T, Snyder HM, Sugimoto T et al: Impact of the COVID-19 pandemic on statistical design and analysis plans for multidomain intervention clinical trials: Experience from World-Wide FINGERS. Alzheimers Dement (N Y) 2021, 7(1):e12143.

18. McDermott MM, Newman AB: Preserving Clinical Trial Integrity During the Coronavirus Pandemic. JAMA 2020, 323(21):21352136.

19. van Dorn A: COVID-19 and readjusting clinical trials. Lancet 2020, 396(10250):523-524.

20. Waterhouse DM, Harvey RD, Hurley P, Levit LA, Kim ES, Klepin HD, Mileham KF, Nowakowski G, Schenkel C, Davis C et al: Early Impact of COVID-19 on the Conduct of Oncology Clinical Trials and Long-Term Opportunities for Transformation: Findings From an American Society of Clinical Oncology Survey. JCO Oncol Pract 2020, 16(7):417-421. 
21. Mitchell EJ, Ahmed K, Breeman S, Cotton S, Constable L, Ferry G, Goodman K, Hickey H, Meakin G, Mironov K et al: It is unprecedented: trial management during the COVID-19 pandemic and beyond. Trials 2020, 21(1):784.

22. Kuderer NM, Choueiri TK, Shah DP, Shyr Y, Rubinstein SM, Rivera DR, Shete S, Hsu CY, Desai A, de Lima Lopes G, Jr. et al: Clinical impact of COVID-19 on patients with cancer (CCC19): a cohort study. Lancet 2020, 395(10241):1907-1918.

23. Mehta V, Goel S, Kabarriti R, Cole D, Goldfinger M, Acuna-Villaorduna A, Pradhan K, Thota R, Reissman S, Sparano JA et al: Case Fatality Rate of Cancer Patients with COVID-19 in a New York Hospital System. Cancer Discov 2020, 10(7):935-941.

24. Li G, Yin C, Zhou Y, Wang T, Chen J, Liu Y, Chen T, Wang H, Zhang L, Chen X: Digitalized Adaptation of Oncology Trials during and after COVID-19. Cancer Cell 2020, 38(2):148-149.

25. Contreras F, Baykal E, Abid G: E-Leadership and Teleworking in Times of COVID-19 and Beyond: What We Know and Where Do We Go. Front Psychol 2020, 11:590271.

26. Kossek EE, Lautsch BA, Eaton SC: Telecommuting, control, and boundary management: Correlates of policy use and practice, job control, and work-family effectiveness. J Vocat Behav 2006, 68(2):347-367.

27. Azarbouyeh A, and Naini, S.: A study on the effect of teleworking on quality of work life.Manage Sci Lett 2014, 4(6):1063-1068.

\section{Figures}

A
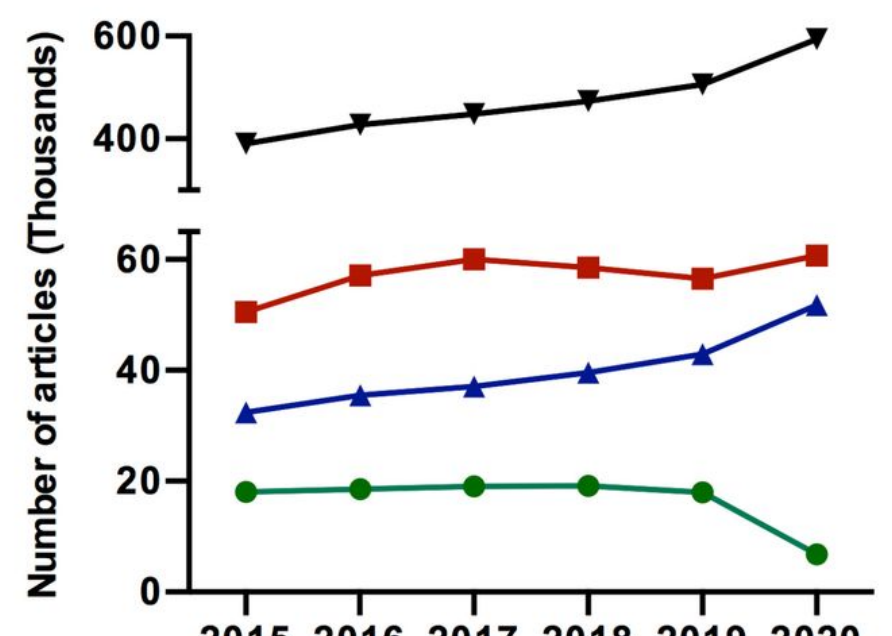

$\Delta$ Original research

- Review

- Clinical Trial

$\rightarrow$ Total

B

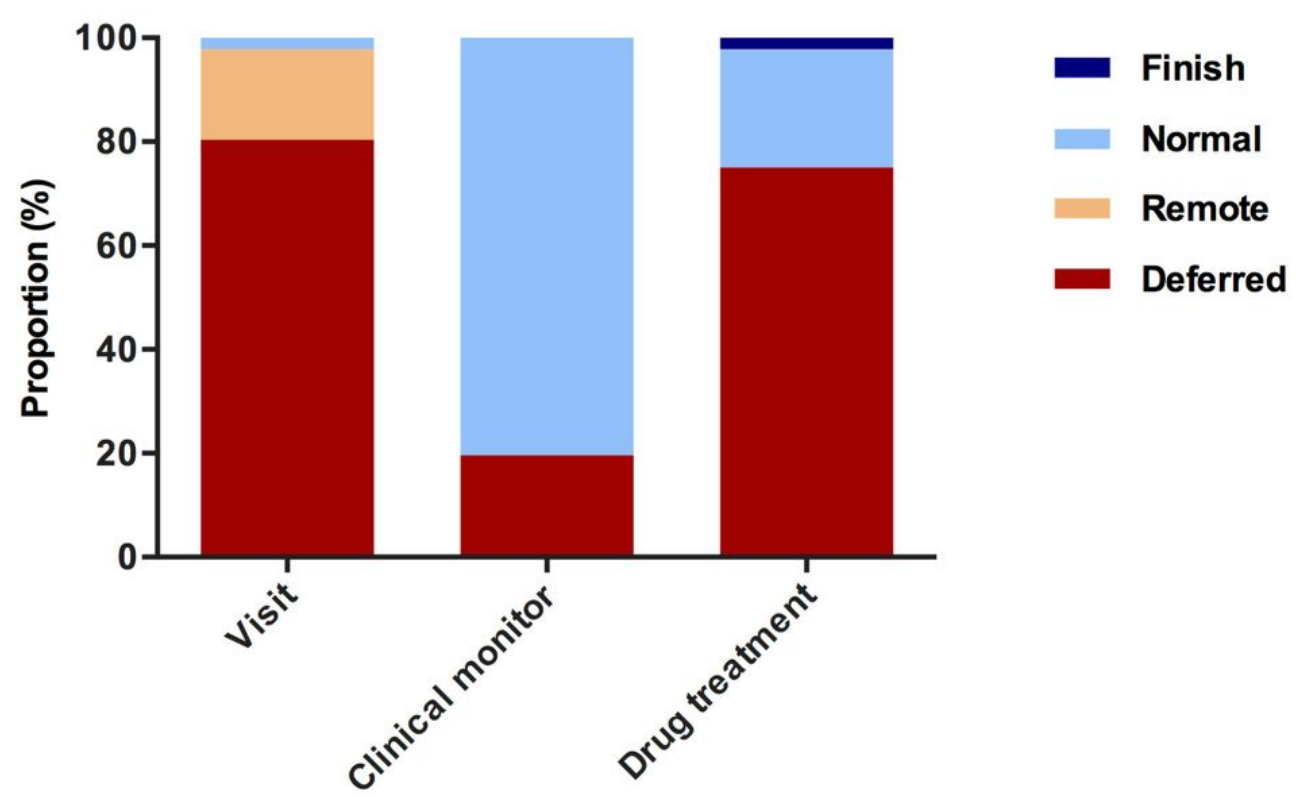


Figure 1

Published paper trends according to types and clinical trials in the Cancer Center of Tongji Hospital in 2020. A. From 2015-2020, the number of published papers transformed according to type. The four lines with various colors and node shapes represent four types of papers. B. Impact of the coronavirus disease pandemic on clinical trials in the Cancer Center of Tongji Hospital in 2020. The bar chart displays the percentages of various outcomes of the clinical trials. The x-axis denotes the three phases of clinical trials.

A



B

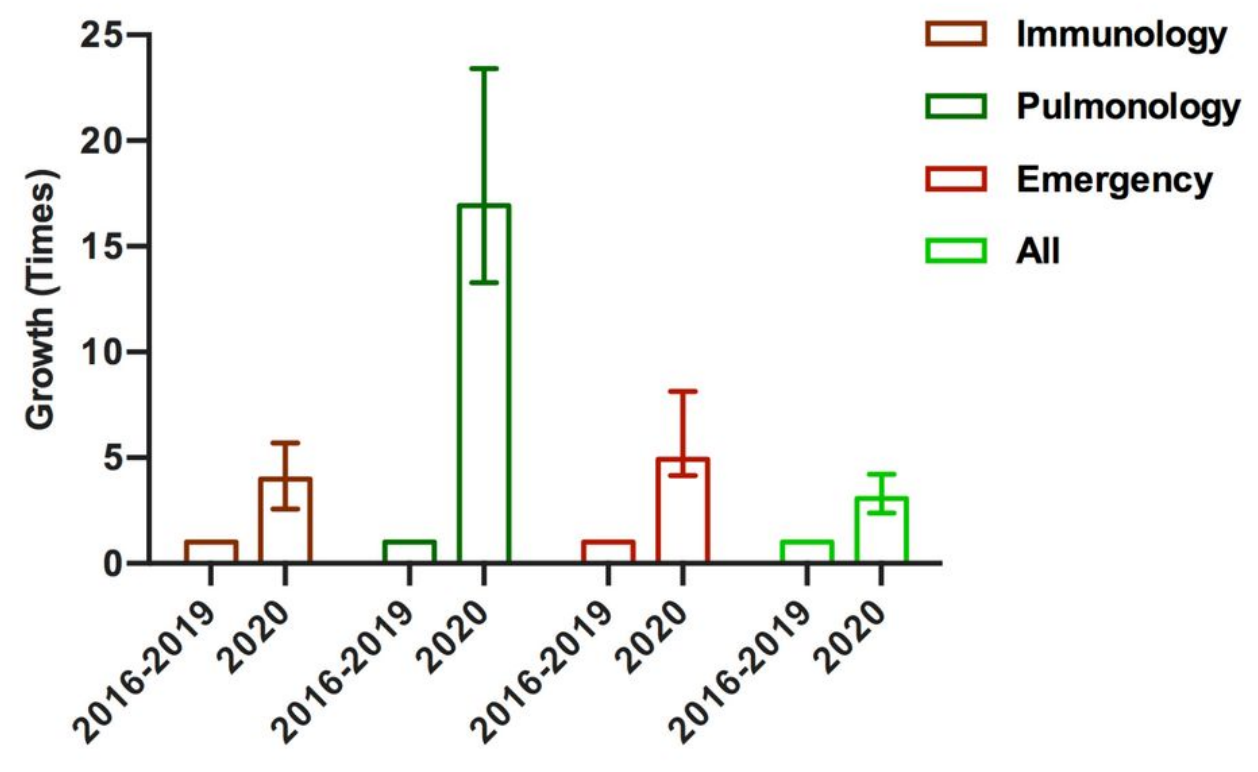

Figure 2

Comparison of the publication growth rates of papers published between 2015-2019 and 2020. A. Compared with the articles per month during 2015 and 2020, the growth of total articles published in 2020/2019 increased compared with the growth of the number of articles published in 2019/2018 ( $P<0.01)$. The others had no statistical significance except for 2016 to $2017(P<0.05)$. B. The bar chart represents the increase in publications. The number of articles published in 2020 is shown relative to 2015-2019. Error bars indicate the maximum and minimum values. ${ }^{*}<0.05, * * P<0.01$. 